\title{
A UNIQUENESS RESULT FOR A SEMILINEAR REACTION-DIFFUSION SYSTEM
}

\author{
M. ESCOBEDO AND M. A. HERRERO \\ (Communicated by Barbara L. Keyfitz)
}

Abstract. Let $(u(t, x), v(t, x))$ and $(\bar{u}(t, x), \bar{v}(t, x))$ be two nonnegative classical solutions of

$$
\begin{cases}u_{t}=\Delta u+v^{p}, & p>0 \\ v_{t}=\Delta v+u^{q}, & q>0\end{cases}
$$

in some strip $S_{T}=(0, T) \times \mathbb{R}^{N}$, where $0<T \leq \infty$, and suppose that

$$
u(0, x)=\bar{u}(0, x), \quad v(0, x)=\bar{v}(0, x),
$$

where $u(0, x)$ and $v(0, x)$ are continuous, nonnegative, and bounded real functions, one of which is not identically zero. Then one has

$$
u(t, x)=\bar{u}(t, x), \quad v(t, x)=\bar{v}(t, x) \quad \text { in } S_{T} .
$$

If $p q \geq 1$, the result is also true if $u(0, x)=v(0, x)=0$. On the other hand, when $0<p q<1$, the set of solutions of (S) with zero initial values is given by

$$
u(t ; s)=c_{1}(t-s)_{+}^{(p+1) /(1-p q)}, \quad v(t ; s)=c_{2}(t-s)_{+}^{(q+1) /(1-p q)},
$$

where $0 \leq s \leq t, c_{1}$ and $c_{2}$ are two positive constants depending only on $p$ and $q$, and $(\xi)_{+}=\max \{\xi, 0\}$.

\section{INTRODUCTION}

In this article we shall concern ourselves with the following initial-value problem:

$$
\begin{array}{cc}
u_{t}=\Delta u+v^{p} ; & t>0, x \in \mathbb{R}^{N} \\
v_{t}=\Delta v+u^{q} ; & t>0, x \in \mathbb{R}^{N},
\end{array}
$$

with $N \geq 1, p>0, q>0$ and

$$
\begin{array}{cc}
u(0, x)=u_{0}(x), & x \in \mathbb{R}^{N} \\
v(0, x)=v_{0}(x), & x \in \mathbb{R}^{N},
\end{array}
$$

Received by the editors November 30, 1989 and, in revised form, March 1, 1990. 1980 Mathematics Subject Classification (1985 Revision). Primary 35K55, 35K57.

Key words and phrases. Reaction diffusion systems, uniqueness.

The first author's work was partially supported by Basque Government Grant G.V. 127310-1/87. The second author's work was partially supported by CICYT Grant PB86-0112-C0202. 
where $u_{0}(x)$ and $v_{0}(x)$ are nonnegative, continuous, and bounded real functions. Equations (1.1) represent a simple example of a reaction-diffusion system, and they can be viewed as a model to describe heat propagation in a two-component combustible mixture. System $(1.1)$ has been analyzed by several authors in the case of bounded and unbounded domains (cf. [7], [8], [5], [4], [3], etc.). In particular, it has been shown in [3] that problem (1.1), (1.2) always has a classical solution in some strip $S_{T}=[0, T) \times \mathbb{R}^{N}$ with $0<T \leq \infty$. By this we shall mean a pair of nonnegative functions, often denoted in the abridged way $(u(t), v(t))$, such that they belong to $C^{2,1}\left(S_{T}\right)$, satisfy (1.1) and (1.2), and remain bounded in any closed strip $\bar{S}_{\tau}=[0, \tau] \times \mathbb{R}^{N}$ with $0<\tau<T$. A number of properties of solutions of (1.1), (1.2) were derived in [3]. In particular (cf. [3, Theorems 2 and 3]) if $p q>1$ and

$$
\frac{\gamma+1}{p q-1} \geq \frac{N}{2}
$$

where $\gamma=\max \{p, q\}$, every nontrivial solution blows up in finite time, in the sense that it becomes unbounded at some $t=T^{*}<+\infty$. On the other hand, if $p q>1$ and (1.3) fails, solutions might be bounded in any strip $S_{T}$ or have a finite blow-up time, according to the size of their initial values $u_{0}, v_{0}$. To complete this picture, let us point out that solutions are global in time if $0<p q \leq 1$ (cf. [3, Theorem 1]).

For the scalar equation

$$
u_{t}=\Delta u+u^{p} ; \quad t>0, \quad x \in \mathbb{R}^{N},
$$

it is well known that there is a finite-time blow-up of nontrivial solutions if

$$
1<p \leq 1+\frac{2}{N} \text {, }
$$

whereas global continuation and blow-up are both possible if $p>1+\frac{2}{N}$ (cf., for instance, [8], [2], [9]). Indeed, every solution is global if $0<p \leq 1$. Notice that (1.3) coincides with (1.5) when $p=q$, in which case (1.1), (1.2) reduce to the Cauchy problem for (1.4) if $u_{0}=v_{0}$.

As recalled in [3], local existence for problem (1.1), (1.2) is rather standard, as can be seen by, say, fixed-point arguments. Uniqueness, however, is not a priori clear except in the straightforward case $p \geq 1$ and $q \geq 1$, and was left open in that paper. For instance, one readily checks that, if $0<p q<1$, functions

$$
u_{1}(t)=c_{1} t^{\alpha} \quad \text { with } \alpha=\frac{p+1}{1-p q}, \quad c_{1}^{1-p q}=(1-p q)^{p+1}(p+1)^{-1}(q+1)^{-p}
$$

and

$$
v_{1}(t)=c_{2} t^{\beta} \quad \text { with } \beta=\frac{q+1}{1-p q}, \quad c_{2} \beta=c_{1}^{q}
$$

solve (1.1) and are such that $u_{1}(0)=v_{1}(0)=0$. We show here the following 
result:

Theorem. Assume that $p$ and $q$ are different from zero and $p<1$ or $q<1$. Then

(a) If $0<p q<1$ and $\left(u_{0}, v_{0}\right) \neq(0,0)$, problem (1.1), (1.2) has a unique solution.

(b) If $0<p q<1$ and $\left(u_{0}, v_{0}\right)=(0,0)$, the set of nontrivial nonnegative solutions of (1.1), (1.2) is given by

$$
u(t ; s)=c_{1}(t-s)_{+}^{\alpha}, \quad v(t ; s)=c_{2}(t-s)_{+}^{\beta},
$$

where $(r)_{+}=\max \{r, 0\}, s$ is any nonnegative real constant, and $c_{1}, c_{2}$, $\alpha$, and $\beta$ are as in (1.6).

(c) If $p q \geq 1$, there is a unique solution of (1.1), (1.2).

\section{Proof OF THE THEOREM}

We begin by recalling an auxiliary result already proved in [3, Lemma 2.4]:

Lemma 1. Let $\left(u_{0}, v_{0}\right) \neq(0,0)$, and let $(u(t, x), v(t, x))$ be a solution of (1.1), (1.2). Then for any $\tau>0$ there exist constants $c>0$ and $\alpha>0$ such that

$$
\begin{aligned}
& u(\tau, x) \geq c \exp \left(-\alpha|x|^{2}\right), \\
& v(\tau, x) \geq c \exp \left(-\alpha|x|^{2}\right) .
\end{aligned}
$$

Proof. We just sketch, for completeness, the case where $u_{0} \neq 0$ and $q \geq 1$. Solutions of (1.1), (1.2) satisfy

$$
\begin{gathered}
u(t)=S(t) u_{0}+\int_{0}^{t} S(t-s) v^{p}(s) d s \\
v(t)=S(t) v_{0}+\int_{0}^{t} S(t-s) u^{q}(s) d s
\end{gathered}
$$

where

$$
S(t) f \equiv S(t) f(x)=(4 \pi t)^{-N / 2} \int_{\mathbb{R}^{N}} \exp \left(-\frac{|x-\xi|^{2}}{4 t}\right) f(\xi) d \xi .
$$

We may assume without loss of generality that $u_{0}>0$ in some ball centered at the origin. Then there exists $R>0$ such that $\nu=\inf \left\{u_{0}(\xi):|\tau| \leq R\right\}>0$ and, by $(2.2 \mathrm{a})$ and $(2.3)$,

$$
u(t, x) \geq S(t) u_{0} \geq \nu \exp \left(-\frac{|x|^{2}}{2 t}\right)(4 \pi \tau)^{-N / 2} \int_{|y| \leq R} \exp \left(-\frac{|y|^{2}}{2 t}\right) d y,
$$

whence (2.1a) with $\alpha=\frac{1}{2 \tau}$ and $c=\nu(4 \pi \tau)^{-N / 2} \int_{|y| \leq R}\left(-\frac{|y|^{2}}{2 \tau}\right) d y$. On the other hand, using (2.2b) and Jensen's inequality,

$$
\begin{aligned}
v(t, x) & \geq \int_{0}^{t} S(t-s)\left(S(s) u_{0}\right)^{q} d s \geq \int_{0}^{t}\left(S(t-s) S(s) u_{0}\right)^{q} d s \\
& =\int_{0}^{t}\left(S(t) u_{0}\right)^{q} d s,
\end{aligned}
$$


whence

$$
v(t, x) \geq t\left(S(t) u_{0}\right)^{q} .
$$

From this (2.1b) follows, with perhaps a different choice of $c$ and $\alpha$.

We now specialize to the case $0<p q<1$.

Lemma 2. Let $(u(t, x), v(t, x))$ be a nontrivial solution of $(1.1)$ with $0<p q<$ 1. Then

$$
\begin{gathered}
u(t, x) \geq c_{1} t^{\alpha} \\
v(t, x) \geq c_{2} t^{\beta},
\end{gathered}
$$

where $c_{1}, c_{2}, \alpha$, and $\beta$ are given in (1.6).

Proof. Assume first that $u_{0}(x) \geq c \exp \left(-\alpha|x|^{2}\right)$ for some $c>0$ and $\alpha>0$. Suppose, for definiteness, that $0<p, q<1$. Since

$$
S(t) \exp \left(-\alpha|x|^{2}\right)=(1+4 \alpha t)^{-N / 2} \exp \left(-\frac{\alpha|x|^{2}}{1+4 \alpha t}\right),
$$

it follows that

$$
u(t, x) \geq S(t) u_{0} \geq c(1+4 \alpha t)^{-N / 2} \exp \left(-\frac{\alpha|x|^{2}}{1+4 \alpha t}\right)
$$

and, by $(2.2 b)$,

$$
v(t, x) \geq \int_{0}^{t} S(t-s)(u(s, x))^{q} d s,
$$

whence

$$
\begin{aligned}
v(t, x) \geq & c^{q} \int_{0}^{t}(1+4 \alpha s)^{-N / 2(q-1)}(1+4 \alpha s+4 \alpha q(t-s))^{-N / 2} \\
& \times \exp \left(-\frac{\alpha q|x|^{2}}{1+4 \alpha s+4 \alpha q(t-s)}\right) d s \\
\geq & c^{q}(1+4 \alpha t)^{-N / 2} \exp \left(-\frac{\alpha q|x|^{2}}{1+4 \alpha q t}\right) t .
\end{aligned}
$$

We substitute this inequality in (2.2a) and use (2.6) to get

$$
\begin{aligned}
u(t, x) \geq & c^{p q} \int_{0}^{t}(1+4 \alpha s)^{-N p / 2} s^{p}(1+4 \alpha q s+4 \alpha p q(t-s))^{-N / 2}(1+4 \alpha q s)^{N / 2} \\
& \times \exp \left(-\frac{\alpha p q|x|^{2}}{1+4 \alpha q s+4 \alpha p q(t-s)}\right) d s,
\end{aligned}
$$

whence

$$
u(t, x) \geq c^{p q}(1+4 \alpha t)^{-N p / 2}(1+4 \alpha q t)^{-N / 2} \exp \left(-\frac{\alpha p q|x|^{2}}{1+4 \alpha p q t}\right) \frac{t^{p+1}}{p+1}
$$


By induction, we obtain, for $j=0,1,2, \ldots$,

$$
\begin{aligned}
u(t, x) \geq & c^{(p q)^{j+1}} f_{j}(t) \exp \left(-\frac{\alpha(p q)^{j+1}|x|^{2}}{1+4 \alpha(p q)^{j+1} t}\right) B_{j+1} A_{j+1} t^{(p+1)} \\
& \times\left[(p q)^{j}+(p q)^{j-1}+\cdots+p q+1\right]
\end{aligned}
$$

where

$(2.8 a)$

$$
\begin{aligned}
f_{j}(t)= & (1+4 \alpha t)^{-(N / 2) p^{j+1} q^{j}}(1+4 \alpha q t)^{-(N / 2) p^{j} q^{j}}(1+4 \alpha p q t)^{-(N / 2) p^{j} q^{j-1}} \\
& \ldots\left(1+4 \alpha p^{j} q^{j+1} t\right)^{-N / 2}
\end{aligned}
$$

$$
\begin{aligned}
B_{j+1}= & \left(\frac{1}{p+1}\right)^{(p q)^{j}}\left(\frac{1}{(p+1)(p q+1)}\right)(p q)^{j-1} \\
& \ldots\left(\frac{1}{(p+1)\left((p q)^{j}+(p q)^{j-1}+\cdots+1\right)}\right)
\end{aligned}
$$

(2.8c) $A_{j+1}=\left[\left(\frac{1}{(p+1)(q+1)}\right)^{q^{j-1} p^{j-1}}\left(\frac{1}{((p+1)(p q+1) q+1)}\right)^{q^{j-2} p^{j-2}}\right.$

$$
\left.\cdots\left(\frac{1}{\left((p+1)\left((p q)^{j-1}+(p q)^{j-2}+\cdots+1\right) q+1\right)}\right)\right]^{p} \text {. }
$$

Notice that

$$
\begin{aligned}
B_{j+1} & =\left(\frac{1}{p+1}\right)^{\left(1-(p q)^{j+1}\right) /(1-p q)}\left[(1+p q)^{-(p q)^{j-1}} \cdots\left((p q)^{j}+(p q)^{j-1}+\cdots+1\right)^{-1}\right] \\
& \geq\left(\frac{1}{p+1}\right)^{1 /(1-p q)}(1-p q)^{1 /(1-p q)}
\end{aligned}
$$

whereas

$$
\begin{aligned}
A_{j+1} & =\left(\prod_{k=0}^{j-1}\left((p+1)\left((p q)^{k}+(p q)^{k-1}+\cdots+p q+1\right) q+1\right)^{-(p q)^{j-1-k}}\right)^{p} \\
& =\left(\prod_{k=0}^{j-1}\left((p+1)\left(\frac{1-(p q)^{k+1}}{1-p q}\right) q+1\right)^{-(p q)^{j-1-k}}\right)^{p} \\
& \geq\left(\prod_{k=0}^{j-1}\left(\frac{(p+1) q}{1-p q}+1\right)^{-(p q)^{j-1-k}}\right)^{p}=\left(\prod_{k=0}^{j-1}\left(\frac{q+1}{1-p q}\right)^{-(p q)^{j-1-k}}\right)^{p},
\end{aligned}
$$

so that

$$
A_{j+1} \geq\left(\frac{1-p q}{q+1}\right)^{p /(1-p q)}
$$


Finally,

$$
\begin{aligned}
f_{j}(t) & =\left(\prod_{k=0}^{j}\left(1+4 \alpha(p q)^{k} t\right)^{-(N / 2) p(p q)^{j-k}}\right)\left(\prod_{k=0}^{j}\left(1+4 \alpha p^{k} q^{k+1} t\right)^{-(N / 2)(p q)^{j-k}}\right) \\
& \equiv\left(f_{j}^{1}(t)\right)\left(f_{j}^{2}(t)\right)
\end{aligned}
$$

and, since

$$
4 \alpha t(p q)^{j}(j+1)=\sum_{k=0}^{j}(p q)^{j-k} 4 \alpha(p q)^{k} t \geq \sum_{k=0}^{j}(p q)^{j-k} \log \left(1+4 \alpha(p q)^{k} t\right),
$$

we deduce that

$$
\lim _{j \rightarrow \infty} f_{j}^{(i)}(t) \geq 1 \text { for } i=1,2 .
$$

Taking into account (2.8)-(2.11), we let $j \rightarrow \infty$ in (2.7) to obtain (2.5a) under our current assumptions. Estimate $(2.5 \mathrm{~b})$ can be obtained in an analogous way. The case where $p$ or $q$ are larger than or equal to 1 is similarly dealt with.

As to the general case, we take $\varepsilon>0$ but otherwise arbitrary, and set $u_{\varepsilon}(t) \equiv$ $u(t+\varepsilon)$. One then has

$$
u_{\varepsilon}(t)=S(t) u_{\varepsilon}(0)+\int_{0}^{t} S(t-s) v_{\varepsilon}^{p}(s) d s,
$$

where, by Lemma $1, u_{\varepsilon}(0) \geq c \exp \left(-\alpha|x|^{2}\right)$ for some $c$ and $\alpha$. Therefore $u_{\varepsilon}(t) \geq c_{1} t^{\alpha}$, and accordingly

$$
u(t)=u(\varepsilon+(t-\varepsilon)) \geq c_{1}(t-\varepsilon)^{\alpha},
$$

whence the result, since $\varepsilon>0$ is arbitrary.

As a next step, we show

Lemma 3. Assume that $0<p q<1$, and suppose that $\left(u_{0}, v_{0}\right) \neq(0,0)$. Then there exists at most one solution of (1.1), (1.2).

Proof. Suppose first that $0<p<1$ and $0<q<1$. We shall argue by contradiction, thus assuming that for some $\left(u_{0}, v_{0}\right) \neq(0,0)$ there exist two different solutions $(u(t), v(t))$ and $(\bar{u}(t), \bar{v}(t))$ defined in some strip $S_{T}$. It then follows from (2.2), (2.5), and the mean value theorem that

$$
\begin{aligned}
(u(t)-\bar{u}(t))_{+} & \leq \int_{0}^{t} S(t-s)\left(v^{p}(s)-\bar{v}^{p}(s)\right)_{+} d s \\
& \leq p c_{2}^{p-1} \int_{0}^{t} S(t-s)(v(s)-\bar{v}(s))_{+} s^{(p-1)(q+1) /(1-p q)} d s .
\end{aligned}
$$

In a similar way, we get

$$
(v(t)-\bar{v}(t))_{+} \leq q c_{1}^{q-1} \int_{0}^{t} S(t-s)(u(s)-\bar{u}(s))_{+} s^{(q-1)(p+1) /(1-p q)} d s
$$


From (2.12) and (2.13), it follows that, setting $\|f(t, .)\|_{\infty}=\sup _{x \in \mathbf{R}^{N}}|f(t,)$.$| ,$

$$
\begin{aligned}
& \left\|(u-\bar{u})_{+}(t)\right\|_{\infty} \\
& \leq p q c_{1}^{q-1} c_{2}^{p-1} \int_{0}^{t} s^{(q+1)(p-1) /(1-p q)}\left(\int_{0}^{s} \tau^{(p+1)(q-1) /(1-p q)}\left\|(u-\bar{u})_{+}(\tau)\right\|_{\infty} d \tau\right) d s \\
& =p q(1-p q)^{-2}(p+1)(q+1) \\
& \quad \times \int_{0}^{t} s^{(q+1)(p-1) /(1-p q)}\left(\int_{0}^{s} \tau^{(p+1)(q-1) /(1-p q)}\left\|(u-\bar{u})_{+}(\tau)\right\| d \tau\right) d s .
\end{aligned}
$$

We next show that the integrand above is indeed locally integrable. To this end, we first notice that, since $p$ and $q$ are less than one,

$$
\left\|(u-\bar{u})_{+}(t)\right\| \leq \int_{0}^{t}\left\|(v-\bar{v})_{+}(s)\right\|_{\infty}^{p} d s \leq \int_{0}^{t}\left(\int_{0}^{s}\left\|(u-\bar{u})_{+}(\tau)\right\|_{\infty}^{q} d \tau\right)^{p} d s,
$$

so that

$$
\left\|(u-\bar{u})_{+}(t)\right\|_{\infty} \leq(p+1)^{-(1 /(1-p q))} t^{(p+1) /(1-p q)} .
$$

This implies that the right-hand side in (2.14) is convergent. Moreover, substituting (2.16) in (2.14) yields

$$
\left\|(u-\bar{u})_{+}(t)\right\|_{\infty} \leq p q(p+1)^{-(1 /(1-p q))} t^{(p+1) /(1-p q)} .
$$

We may now use (2.17) to obtain a new bound for $\left\|(u-\bar{u})_{+}(t)\right\|_{\infty}$ via (2.14). Iterating this procedure $k$ times, we obtain

$$
\left\|(u-\bar{u})_{+}(t)\right\|_{\infty} \leq(p q)^{k}(p+1)^{-(1 /(1-p q))} t^{(p+1) /(1-p q)} .
$$

Now letting $k \rightarrow \infty$, it follows that $u \equiv \bar{u}$, whence $v \equiv \bar{v}$.

It remains to consider yet the situation where one of the exponents $p, q$ is larger than or equal to one. Assume for instance that $q>1$, and set $f(s)=s^{p}$, $g(v)=S(s) v_{0}+\int_{0}^{s} S(s-\tau) v^{q}(\tau) d \tau$. Using (2.2) as well as the mean value theorem for $(f \circ g)$, it follows that for some $w=\theta u+(1-\theta) \bar{u}$ with $\theta \equiv \theta(\tau)$ and $0<\theta<1$,

$$
\begin{aligned}
u(t)-\bar{u}(t) \leq & p q \int_{0}^{t} S(t-s)\left(\int_{0}^{s} S(s-\tau) w^{q}(\tau) d \tau\right)^{p-1} \\
& \times\left(\int_{0}^{s} S(s-\tau) w^{q-1}(\tau)(u-\bar{u})(\tau) d \tau\right) d s .
\end{aligned}
$$

On the other hand, by the Hölder inequality,

$$
\begin{aligned}
& \int_{0}^{s} S(s-\tau) w^{q-1}(\tau)(u-\bar{u})(\tau) d \tau \\
& \quad \leq\left(\int_{0}^{s} S(s-\tau)|w(\tau)|^{q} d \tau\right)^{(q-1) / q}\left(\int_{0}^{s} S(s-\tau)|(u-\bar{u})(\tau)|^{q} d \tau\right)^{1 / q}
\end{aligned}
$$


and therefore

$$
\begin{aligned}
\left\|(u-\bar{u})_{+}(t)\right\|_{\infty} \leq & p q \int_{0}^{t}\left(\int_{0}^{s} S(s-\tau)|w(\tau)|^{q} d \tau\right)^{p-1+(q-1) / q} \\
& \times\left(\int_{0}^{s} S(s-\tau)|(u-\bar{u})(\tau)|^{q} d \tau\right)^{1 / q} d s
\end{aligned}
$$

Taking (2.5) into account again, we obtain

$$
\left\|(u-\bar{u})_{+}(t)\right\| \leq c \int_{0}^{t} s^{-(q+1) / q}\left(\int_{0}^{s}\left\|(u-\bar{u})_{+}(\tau)\right\|_{\infty}^{q} d \tau\right)^{1 / q} d s,
$$

where $c=p q(1-p q)^{-(q+1) / q}(q+1)^{1 / q}(p+1)$. We now claim that

$$
v^{p}(t)-\bar{v}^{p}(t) \leq\left(\int_{0}^{t} S(t-s)(u-\bar{u})_{+}^{q}(s) d s\right)^{p} .
$$

Assuming this inequality for the moment, the proof is complete, since (2.12) implies that

$$
\left\|(u-\bar{u})_{+}(t)\right\|_{\infty} \leq \int_{0}^{t}\left\|v^{p}(s)-\bar{v}^{p}(s)\right\|_{\infty} d s \leq \int_{0}^{t}\left(\int_{0}^{s}\left\|(u-\bar{u})_{+}(\tau)\right\|_{\infty}^{q} d \tau\right)^{p} d s,
$$

so that (2.16) holds, and the result follows at once from (2.20) and (2.16), as in the previous case. To show (2.21), let us write

$$
K_{t}(x)=(4 \pi t)^{-N / 2} \exp \left(-\frac{|x|^{2}}{4 t}\right) .
$$

For arbitrary $\alpha \in(0,1)$, one then has

$$
\begin{aligned}
v(t) & =S(t) v_{0}+\int_{0}^{t} S(t-s) u^{q}(s) d s=S(t) v_{0}+\int_{0}^{t} \int_{\mathbb{R}^{N}} K_{t-s}(x-y) u^{q}(s, y) d y d s \\
& =S(t) v_{0}+\int_{0}^{t} \int_{\mathbb{R}^{N}} K_{t-s}^{(q-\alpha) / q}(x-y) u^{q-\alpha}(s, y) K_{t-s}^{\alpha / q}(x-y) u^{\alpha}(s, y) d y d s .
\end{aligned}
$$

Since $u=\bar{u}+(u-\bar{u}) \leq \bar{u}+(u-\bar{u})_{+}$and $u^{\alpha} \leq \bar{u}^{\alpha}+(u-\bar{u})_{+}^{\alpha}$, we have

$$
\begin{aligned}
v(t) \leq & S(t) v_{0}+\int_{0}^{t} \int_{\mathbb{R}^{N}} K_{t-s}^{(q-\alpha) / q}(x-y) u^{q-\alpha}(s, y) \cdot K_{t-s}^{\alpha / q}(x-y) \bar{u}^{\alpha}(s, y) d y d s \\
& +\int_{0}^{t} \int_{\mathbb{R}^{N}} K_{t-s}^{(q-\alpha) / q}(x-y) u^{q-\alpha}(s, y) K_{t-s}^{\alpha / q}(x-y)(u-\bar{u})_{+}^{\alpha}(s, y) d y d s .
\end{aligned}
$$


Using Hölder's inequality, we obtain

$$
\begin{aligned}
v(t) \leq & S(t) v_{0}+\int_{0}^{t}\left(\int_{\mathbb{R}^{N}} K_{t-s}(x-y) u^{q}(s, y) d y\right)^{(q-\alpha) / q} \\
& \times\left(\int_{\mathbb{R}^{N}} K_{t-s}(x-y) \bar{u}^{q}(s, y) d y\right)^{\alpha / q} d s \\
& +\int_{0}^{t}\left(\int_{\mathbb{R}^{N}} K_{t-s}(x-y) u^{q}(s, y) d y\right)^{(q-\alpha) / q} \\
& \times\left(\int_{\mathbb{R}^{N}} K_{t-s}(x-y)(u-\bar{u})_{+}^{q}(s, y) d y\right)^{\alpha / q} d s \\
\leq & S(t) v_{0}+\left(\int_{0}^{t} S(t-s) u^{q}(s) d s\right)^{(q-\alpha) / q} \\
& \times\left(\int_{0}^{t} S(t-s) \bar{u}^{q}(s) d s\right)^{\alpha / q} \\
& +\left(\int_{0}^{t} S(t-s) u^{q}(s) d s\right)^{(q-\alpha) / q}\left(\int_{0}^{t} S(t-s)(u-\bar{u})_{+}^{q}(s) d s\right)^{\alpha / q},
\end{aligned}
$$

so that, using the fact that for any nonnegative $a, b$, and $c$ and any $\theta \in(0,1)$,

$$
a+b^{1-\theta} c^{\theta} \leq(a+b)^{1-\theta}(a+c)^{\theta},
$$

one arrives at

$$
v(t) \leq v(t)^{(q-\alpha) / q} \bar{v}(t)^{\alpha / q}+v(t)^{(q-\alpha) / q}\left(\int_{0}^{t} S(t-s)(u-\bar{u})_{+}^{q}(s) d s\right)^{\alpha / q}
$$

and, setting $\alpha=p q,(2.21)$ follows.

Concerning the case where $u_{0}=v_{0}=0$, we have the following lemma:

Lemma 4. Assume that $0<p q<1$ and $u_{0}=v_{0}=0$. The set of nontrivial nonnegative solutions of (1.1), (1.2) is then given by the family

$$
u(t ; s)=c_{1}(t-s)_{+}^{(p+1) /(1-p q)}, \quad v(t ; s)=c_{2}(t-s)_{+}^{(q+1) /(1-p q)},
$$

where $0 \leq s<t$ and $c_{1}, c_{2}$ are the constants in (1.6).

Proof. The proof consists of a slight modification of the corresponding result for the scalar equation (1.4) (cf. [1]). Let $(u, v)$ be a nontrivial solution of (1.1), (1.2) under our current hypotheses. Then, by (2.2)

$$
\|u(t)\|_{\infty} \leq \int_{0}^{t}\left(\int_{0}^{s}\|u(\tau)\|_{\infty}^{q} d \tau\right)^{p} d s,\|v(t)\|_{\infty} \leq \int_{0}^{t}\left(\int_{0}^{s}\|v(\tau)\|_{\infty}^{p} d \tau\right)^{q} d s,
$$

so that

$$
\|u(t)\|_{\infty} \leq c_{1} t^{(p+1) /(1-p q)}, \quad\|v(t)\|_{\infty} \leq c_{2} t^{(q+1) /(1-p q)}
$$


with $c_{1}, c_{2}$ as in (1.6). By hypothesis, there exist $t>0$ and $x \in \mathbb{R}^{N}$ such that $u(t, x)>0$ or $v(t, x)>0$. Assume, for definiteness, that $u(t, x)>0$, and define $\tau$ as follows

$$
\tau=\inf \{t>0: u(t, x)>0\} .
$$

By standard results, $u(t, x)>0$ and $v(t, x)>0$ for any $x \in \mathbb{R}^{N}$ and $t>\tau$. Now take $\bar{t}>\tau$ and set

$$
\bar{u}(t, x)=u(t+\bar{t}, x), \quad \bar{v}(t, x)=v(t+\bar{t}, x) .
$$

Then $(\bar{u}, \bar{v})$ solves $(1.1)$ and $\bar{u}(0, x)>0, \bar{v}(0, x)>0$. Therefore, by Lemma 2,

$$
u(t+\bar{t}, x) \geq c_{1} t^{(p+1) /(1-p q)}, \quad v(t+\bar{t}, x) \geq c_{2} t^{(q+1) /(1-p q)}
$$

for any $t \geq 0$. This implies that

$$
u(t, x) \geq c_{1}(t-\tau)_{+}^{(p+1) /(1-p q)}, \quad v(t, x) \geq c_{2}(t-\tau)_{+}^{(q+1) /(1-p q)}
$$

for $x \in \mathbb{R}^{N}, t \geq 0$. Now choose $\underline{t}<\tau$ and define

$$
\underline{u}(t, x)=u(t+\underline{t}, x), \quad \underline{v}(t, x)=v(t+\underline{t}, x) .
$$

By our choice of $\tau,(\underline{u}, \underline{v})$ solves $(1.1),(1.2)$ with $\underline{u}(0)=\underline{v}(0)=0$. Therefore, by $(2.22)$

$$
u(t+\underline{t}, x) \leq c_{1} t^{p+1 / 1-p q}, \quad v(t+\underline{t}, x) \leq c_{2} t^{(q+1) /(1-p q)}
$$

for any $t \geq 0$ and $x \in \mathbb{R}^{N}$, and this implies

$$
u(t, x) \leq c_{1}(t-\tau)_{+}^{(p+1) /(1-p q)}, \quad v(t, x) \leq c_{2}(t-\tau)_{+}^{(q+1) /(1-p q)}
$$

for $x \in \mathbb{R}^{N}, t \geq 0$, and the conclusion follows from (2.22) and (2.23).

Our last step consists of this lemma:

Lemma 5. Let $p>0$ and $q>0$ be such that $p q \geq 1$. Then, if $(u(t), v(t))$ and $(\bar{u}(t), \bar{v}(t))$ are solutions of $(1.1),(1.2)$ in some strip $S_{T}$, it follows that $u(t)=\bar{u}(t)$ and $v(t)=\bar{v}(t)$ in $S_{T}$.

Proof. The result is rather classical if $p \geq 1$ and $q \geq 1$. Assume for instance that $0<p<1 \leq q$. As in the proof of Lemma 3, we set $f(s)=s^{p}, g(v)(s)=$ $S(s) v_{0}+\int_{0}^{s} S(s-\tau) v^{q}(\tau) d \tau$ and use the Mean Value Theorem to get (2.19). We now set

$$
F(t)=\sup _{0 \leq s \leq t}\|(u-\bar{u})(s)\|_{\infty}, \quad 0<t<T
$$


Clearly

$$
\begin{aligned}
\int_{0}^{s} S(s-\tau) w^{q-1}(\tau)(u-\bar{u})(\tau) d \tau \\
\quad \leq F(t) \int_{0}^{s} S(s-\tau) w^{q-1}(\tau) d \tau \\
\quad \leq F(t)\left(\int_{0}^{s} S(s-\tau) w^{q}(\tau) d \tau\right)^{(q-1) / q} \\
\quad \leq F(t)\left\|\int_{0}^{s} S(s-\tau) w^{q}(\tau) d \tau\right\|_{\infty}^{(q-1) / q+p-1} \cdot\left(\int_{0}^{s} S(s-\tau) w^{q}(\tau) d \tau\right)^{1-p},
\end{aligned}
$$

and substituting this into (2.19) yields

$$
\|(u-\bar{u})(t)\|_{\infty} \leq p q F(t) \int_{0}^{t} S(t-s)\left\|\int_{0}^{s} S(s-\tau) w^{q}(\tau)\right\|_{\infty}^{p-1 / q} d s .
$$

As $p q \geq 1$, one is thus led to

$$
F(t) \leq K t F(t) \text { for } t \text { small enough, }
$$

for some constant $K$ depending on $p, q, T$ and the bounds on $u$ and $\bar{u}$ in $\mathbb{R}^{N} \times[0, t]$. Since (2.25) implies $F(t) \equiv 0$ for $t$ small enough, the result follows by a suitable iteration of the previous argument. The case $0<q<1 \leq p$ is similar.

\section{REFERENCES}

1. J. Aguirre and M. Escobedo, $A$ Cauchy problem for $u_{t}-\Delta u=u^{p}$ with $0<p<1$ : Asymptotic behaviour of solutions, Ann. Fac. Sci. Toulouse 8 (1986-87), 175-203.

2. D. G. Aronson and H. F. Weinberger, Multidimensional nonlinear diffusion arising in population genetics, Adv. in Math. 30 (1978), 33-76.

3. M. Escobedo and M. A. Herrero, Boundedness and blow-up for a semilinear reaction diffusion system, J. Differential Equations (to appear).

4. M. Floater and J. B. McLeod, in preparation.

5. A. Friedman and Y. Giga, A single point blow up for solutions of semilinear parabolic systems, J. Fac. Sci. Univ. of Tokyo, Sect. I 34 (1987), 65-79.

6. H. Fujita, On the blowing up of solutions of the Cauchy problem for $u_{t}-\Delta u=u^{1+\alpha}$, J. Fac. Sci. Univ. of Tokyo, Sect. I 13 (1960), 109-124.

7. V. A. Galaktionov, S. P. Kurdyumov and A. A. Samarskii, A parabolic system of quasilinear equations I, Differential Equations 19 (1983), 2123-2143.

8. __ A parabolic system of quasilinear equations II, Differential Equations 21 (1985), 15441559.

9. F. B. Weissler, Existence and nonexistence of global solutions for a semilinear heat equation, Israel J. of Math. 38 (1981), 29-40.

Departamento de Matemáticas, facultad de Ciencias, Universidad del Pais Vasco, Apartado 644, 48080 Bilbao, Spain

Departamento de Matemática Aplicada, Facultad de Matemáticas, Universidad ComPLUTENSE, 28040 MADRID, SPAIN 